\title{
Prognostic Value of Pretreatment Controlling Nutritional Status Score for Patients With Pancreatic Cancer: A Meta-Analysis
}

\author{
Xiaofeng Ma, Weihua Zou and Yu Sun * \\ Clinical Laboratory, Huzhou Central Hospital, Affiliated Central Hospital of Huzhou University, Huzhou, China
}

\section{OPEN ACCESS}

Edited by:

Alessio G. Morganti,

University of Bologna, Italy

Reviewed by:

Prof Amit Gupta,

All India Institute of Medical Sciences,

Rishikesh, India

Milly Buwenge,

University of Bologna, Italy

*Correspondence:

Yu Sun

19719792@qq.com

Specialty section:

This article was submitted to Gastrointestinal Cancers: Hepato

Pancreatic Biliary Cancers,

a section of the journal

Frontiers in Oncology

Received: 05 September 2021 Accepted: 06 December 2021

Published: 20 January 2022

Citation:

Ma X, Zou W and Sun Y (2022) Prognostic Value of Pretreatment

Controlling Nutritional Status

Score for Patients With Pancreatic

Cancer: A Meta-Analysis.

Front. Oncol. 11:770894.

doi: 10.3389/fonc.2021.770894
Background: Previous studies have explored the prognostic value of the pretreatment Controlling Nutritional Status (CONUT) score of patients with pancreatic cancer. However, the results of those studies were inconsistent. We used meta-analysis to investigate the impact of the CONUT score on the prognosis for patients with pancreatic cancer.

Methods: We thoroughly searched the PubMed, Web of Science, Embase, and Cochrane Library databases for relevant articles from inception to November 19, 2021. Combined hazard ratios (HRs) and 95\% confidence intervals (95\% Cls) were used to estimate the prognostic value of the CONUT score with respect to survival duration. The pooled odds ratios (ORs) and 95\% Cls were used to estimate the correlation between the CONUT score and clinical characteristics.

Results: The database search found seven studies with 2,294 patients for inclusion in this meta-analysis. A high CONUT score was significantly associated with poor overall survival (OS) $(\mathrm{HR}=1.56,95 \% \mathrm{Cl}=1.13-2.16, p=0.007)$, but not with recurrence-free survival (RFS) (HR $=1.47,95 \% \mathrm{Cl}=0.97-2.23, p=0.072)$ of patients with pancreatic cancer. Moreover, there was a significant association between an elevated CONUT score and male patients $(\mathrm{OR}=1.34,95 \% \mathrm{Cl}=1.03-1.75, p=0.029)$. However, there was no significant association between the CONUT score and the clinical stage (OR $=1.11,95 \%$ $\mathrm{Cl}=0.46-2.71, p=0.576)$, lymph node metastasis $(\mathrm{OR}=0.73,95 \% \mathrm{Cl}=0.39-1.36, p=$ 0.323 ), lymphatic vessel invasion ( $\mathrm{OR}=0.84,95 \% \mathrm{Cl}=0.55-1.28, p=0.411)$, invasion of the portal vein system $(\mathrm{OR}=1.04,95 \% \mathrm{Cl}=0.51-2.13, p=0.915)$, and nerve plexus invasion $(\mathrm{OR}=1.22,95 \% \mathrm{Cl}=0.83-1.80, p=0.318)$ in patients with pancreatic cancer.

Conclusions: The results of our meta-analysis indicate that a high CONUT score predicts a poor OS in patients with pancreatic cancer. The CONUT score may be an effective prognostic factor in pancreatic cancer in clinical practice.

Keywords: CONUT score, pancreatic cancer, meta-analysis, prognostic factors, evidence-based medicine 


\section{INTRODUCTION}

Pancreatic cancer is the seventh most deadly cancer worldwide (1). In 2018, there were 458,918 new cases of pancreatic cancer and 432,242 deaths (1). Pancreatic cancer has a high mortality rate. Although the diagnosis and treatment of pancreatic cancer have greatly advanced over the last several decades, its prognosis remains dismal (2). The 5-year survival rate for all stages of pancreatic cancer is $4.2 \%$ (3). The prognosis for patients with metastatic disease is poor, with a 5-year survival rate of $17.4 \%$ for patients who undergo surgical resection and only $0.9 \%$ for patients who do not undergo resection (3). Therefore, identification of novel and cost-effective biomarkers that can predict the prognosis of patients with pancreatic cancer and provide guidance for individualized treatment is urgently needed.

Increasing evidence has shown that the nutritional status and inflammatory status of the patient play pivotal roles in the development and progression of cancer (4). Many nutritional assessment biomarkers, including the C-reactive protein-toalbumin ratio (5), prognostic nutritional index (6), albumin-toglobulin ratio (7), modified Glasgow prognostic score (8), and the Controlling Nutritional Status (CONUT) score, have been used to predict the prognosis of patients with pancreatic cancer $(9,10)$. In 2005, Ignacio de Ulíbarri first proposed CONUT and used it to evaluate the nutritional status of patients (11). The calculation of the CONUT score is based on serum albumin, total lymphocyte count, and total cholesterol level (11). The CONUT scoring system is shown in Table 1; the score ranges from 0 to 12 . The nutritional status of patients with CONUT scores of $0-1,2-4,5-8$, and 9-12 is normal, light, moderate, and severe, respectively. The higher the CONUT score, the worse the nutritional status. Many studies have investigated the prognostic role of the CONUT score for patients with pancreatic cancer; however, the results of these studies were inconsistent $(9,10,12-16)$. Therefore, we performed this metaanalysis to investigate the prognostic and clinicopathological significance of the CONUT score for patients with pancreatic cancer.

\section{MATERIALS AND METHODS}

\section{Literature Search}

This meta-analysis was performed in accordance with the reporting guidelines of the Preferred Reporting Items for
Systematic Reviews and Meta-Analyses (PRISMA) (17). We thoroughly searched the PubMed, Web of Science, Embase, and Cochrane Library databases for relevant articles from inception to November 19, 2021, using the following search items: "Controlling Nutritional Status", "CONUT", "pancreatic cancer", "pancreatic carcinoma", and "pancreatic neoplasms". All searches were performed using a combination of MeSH terms and free-text words. The publication language was limited to English. References within the identified articles were manually examined to identify other potentially eligible studies.

\section{Inclusion and Exclusion Criteria}

The inclusion criteria for a study were as follows: (1) patients were diagnosed with pancreatic cancer histologically; (2) the study reported the association between the pretreatment CONUT score and all survival outcomes, including but not limited to overall survival (OS), disease-free survival (DFS), and recurrence-free survival (RFS); (3) the hazard ratios (HRs) and $95 \%$ confidence intervals (95\% CIs) were reported in the text or available according to the provided data; (4) a cutoff CONUT score was identified; and (5) the published study was in English. The exclusion criteria were as follows: (1) letters, reviews, comments, case reports, and meeting abstracts; (2) the study did not provide HRs and 95\% CIs for analysis; (3) the study was a duplicate; and (4) the study was not on humans.

\section{Data Extraction and Quality Assessment}

Two investigators (XM and WZ) independently assessed the eligible studies, and all disagreements were resolved by discussion with a third investigator (YS). The following information was extracted from each included study: first author's name, year of publication, sample size, age, histological type, study design, tumor stage, treatment methods, cutoff CONUT score, study period, survival outcomes, survival analysis methods, and HRs and 95\% CIs. The HRs and 95\% CIs from multivariate analysis (MVA) were extracted, if provided; otherwise, the HRs and 95\% CIs from univariate analysis (UVA) were used. MVA considers confounding factors and is more precise than UVA. Two investigators (XM and YS) independently assessed the quality of the eligible studies using the Newcastle-Ottawa Scale (NOS) (18). The NOS evaluates the methodological quality of a study with respect to patient selection, comparability of the study

TABLE 1 | The CONUT scoring system.

\begin{tabular}{|c|c|c|c|c|}
\hline Parameters & \multicolumn{4}{|c|}{ Degree } \\
\hline Serum albumin (g/dL) & $3.5-4.5$ & 3.0-3.49 & $2.5-2.99$ & $<2.50$ \\
\hline Total serum cholesterol (mg/dL) & $\geq 180$ & $140-180$ & $100-139$ & $<100$ \\
\hline Score & 0 & 1 & 2 & 3 \\
\hline Total lymphocyte count $\left(/ \mathrm{mm}^{3}\right)$ & $\geq 1600$ & $1200-1599$ & 800-1199 & $<800$ \\
\hline
\end{tabular}

CONUT, controlling nutritional status. 
groups, and outcome assessment. The maximum NOS score is 9 , so a study with a NOS score of $\geq 6$ was considered high quality.

\section{Statistical Analysis}

The combination of HRs and 95\% CIs was used to estimate the prognostic value of the CONUT score for predicting survival duration. Heterogeneity among the studies in this meta-analysis was evaluated using Cochran's $\mathrm{Q}$ test and the $\mathrm{I}^{2}$ statistical methods. If $\mathrm{I}^{2}>50 \%$ or $p<0.10$, indicating significant heterogeneity among the studies, then a random-effect model was used; otherwise, a fixed-effect model was used. Subgroup analysis, stratified by various factors, was performed to investigate the source of heterogeneity and for further investigations. The pooled odds ratios (ORs) and 95\% CIs were used to estimate the correlation between the CONUT score and the clinical characteristics of pancreatic cancer. Potential publication bias was evaluated using Begg's test with funnel plots and Egger's test. Statistical significance was set at $p<0.05$. Stata software ver. 12.0 (Stata Corporation, College Station, TX, USA) was used for all statistical analyses.

\section{Ethical Statement}

Ethical approval and patient consent were not required because all analyses were based on previously published studies.

\section{RESULTS}

\section{Characteristics of Included Articles}

Seven studies with 2,294 patients $(9,10,12-16)$ were included in this meta-analysis. The procedure used to select the included articles is presented in the flow diagram in Figure 1. The baseline characteristics of the seven included studies are presented in Table 2. Four studies were conducted in Japan $(9,10,13,16)$, and three were performed in China $(12,14,15)$. The sample size ranged from 72 to 589 , with a median of 307 . Six studies were retrospective $(9,10,12-14,16)$, and one was prospective (15). All studies included patients with pancreatic ductal adenocarcinoma (PDAC). Five studies $(10,12,14-16)$ recruited patients who underwent surgery, one study enrolled patients who received chemotherapy (9), and one study enrolled patients who underwent surgery and chemotherapy (13). Four studies (9, 12, $14,16)$ used a cutoff CONUT score of $\geq 3$, two $(10,13)$ used a cutoff CONUT score of $\geq 4$, and one (15) used a cutoff CONUT score of $\geq 2$. All seven studies $(9,10,12-16)$ reported the prognostic role of the CONUT score for predicting OS, and five studies $(9,10,13,14,16)$ presented the prognostic impact of the CONUT score on the RFS. The NOS scores of the seven studies ranged from 7 to 9 , indicating that all included studies were of high quality.

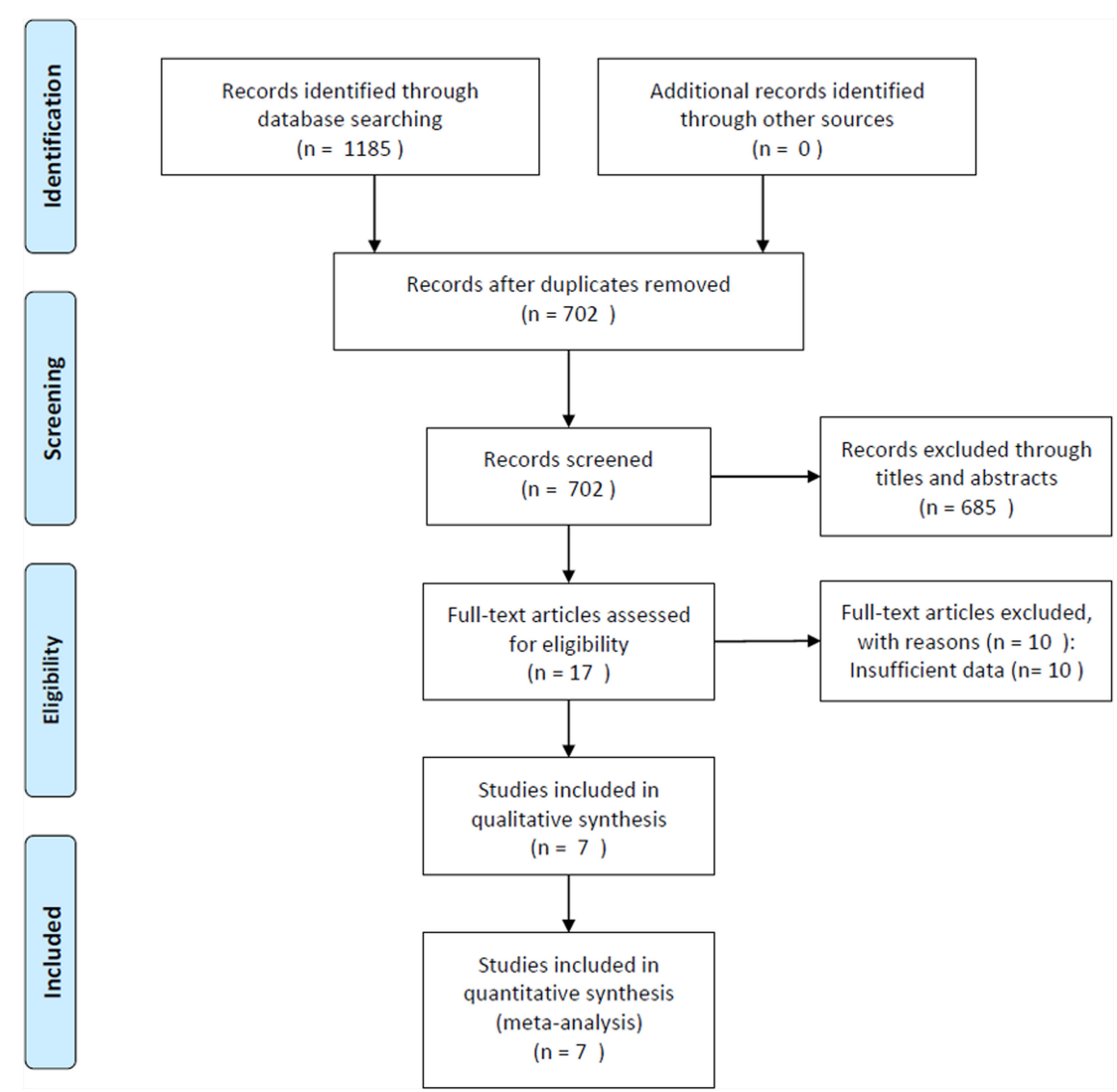

FIGURE 1 | Flowchart of study selection for inclusion in the meta-analysis. 
TABLE 2 | Baseline characteristics of included in this meta-analysis.

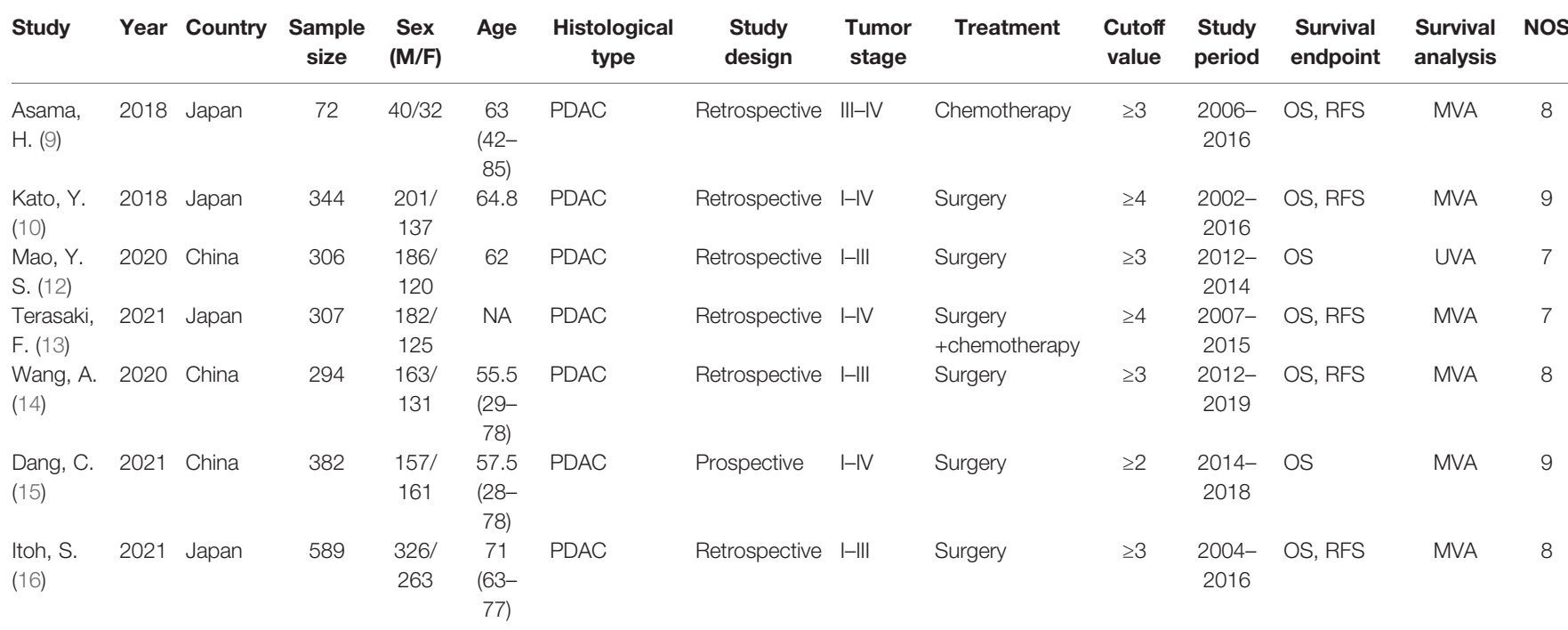

M, male; F, female; PDAC, pancreatic ductal adenocarcinoma; OS, overall survival; RFS, recurrence-free survival; MVA, multivariate analysis; UVA, univariate analysis; NA, not available; NOS, Newcastle-Ottawa Scale.

\section{Prognostic Value of CONUT Score for Predicting OS in Pancreatic Cancer}

The seven included studies of this meta-analysis (9, 10, 12-16) reported the prognostic efficiency of the CONUT score in predicting OS. Because significant heterogeneity $\left(\mathrm{I}^{2}=89.0 \%\right.$, $p<0.001)$ was detected, a random-effect model was used. As shown in Figure 2 and Table 3, the pooled HR and 95\% CI were $\mathrm{HR}=1.56,95 \% \mathrm{CI}=1.13-2.16, p=0.007$, suggesting that a high CONUT score was significantly associated with a poor OS for patients with pancreatic cancer. Subgroup analysis found that an elevated CONUT score $(\mathrm{HR}=1.67,95 \% \mathrm{CI}=1.15-2.41, p=$ 0.007 ) predicted a poor OS for patients undergoing surgery. Additionally, a cutoff CONUT score of $\geq 4(\mathrm{HR}=1.67,95 \% \mathrm{CI}=$
1.26-2.20, $p<0.001$ ) predicted a poor OS for patients with pancreatic cancer (Table 3 ).

\section{Prognostic Value of CONUT Score for Predicting RFS in Pancreatic Cancer}

Five studies consisting of 1,606 patients $(9,10,13,14,16)$ investigated the prognostic value of the CONUT score in predicting RFS. As shown in Figure $\mathbf{3}$ and Table 3, an elevated CONUT score was not significantly associated with the RFS of patients with pancreatic cancer $(\mathrm{HR}=1.47,95 \%$ $\mathrm{CI}=0.97-2.23, p=0.072)$. A random-effect model was used because of significant heterogeneity $\left(\mathrm{I}^{2}=85.6 \%, p<0.001\right)$. Subgroup analysis showed that a high CONUT score predicted

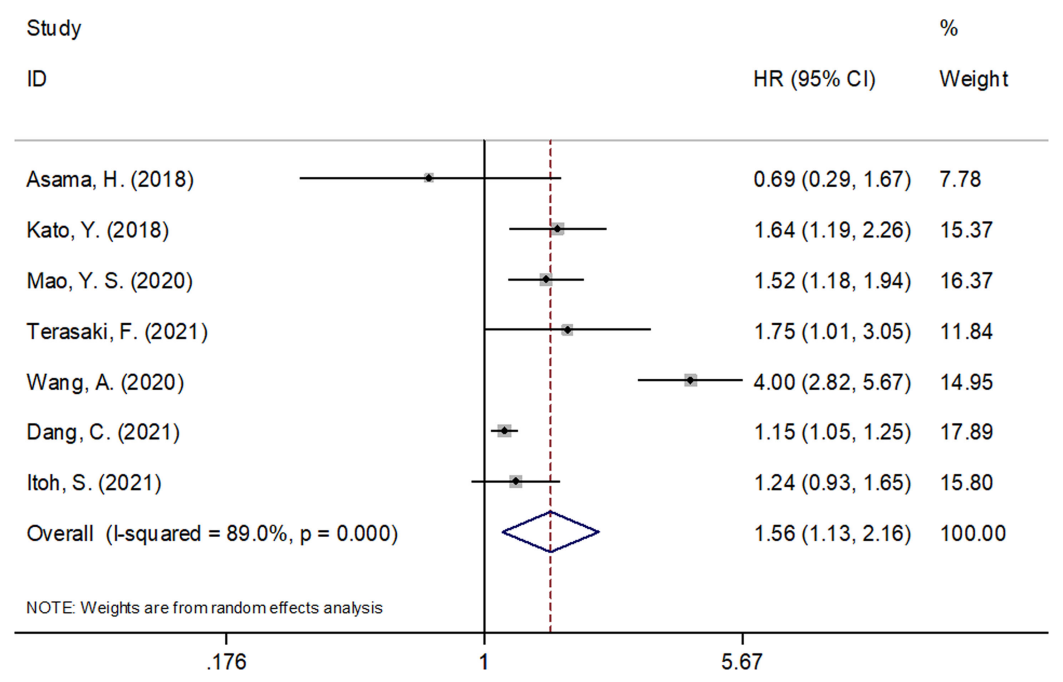

FIGURE 2 | Forest plots of CONUT score in predicting OS in pancreatic cancer. 
TABLE 3 | The prognostic value of COUNT score for OS and RFS in pancreatic cancer and subgroup analysis.

\begin{tabular}{|c|c|c|c|c|c|c|c|}
\hline \multirow[t]{2}{*}{ Variables } & \multirow[t]{2}{*}{ No. of studies } & \multirow[t]{2}{*}{ No. of patients } & \multirow[t]{2}{*}{ HR (95\% Cl) } & \multirow[t]{2}{*}{$\mathbf{p}$} & \multicolumn{2}{|c|}{ Heterogeneity } & \multirow[t]{2}{*}{ Effects model } \\
\hline & & & & & $I^{2}(\%)$ & $\mathbf{P h}$ & \\
\hline \multicolumn{8}{|l|}{ Overall survival } \\
\hline Total & 7 & 2,294 & $1.56(1.13-2.16)$ & 0.007 & 89.0 & $<0.001$ & Random \\
\hline \multicolumn{8}{|l|}{ Country } \\
\hline Japan & 4 & 1,312 & $1.39(1.15-1.69)$ & 0.001 & 37.2 & 0.189 & Fixed \\
\hline China & 3 & 982 & $1.87(1.01-3.47)$ & 0.048 & 95.9 & $<0.001$ & Random \\
\hline \multicolumn{8}{|l|}{ Sample size } \\
\hline$<330$ & 3 & 685 & $1.74(0.75-4.02)$ & 0.198 & 92.2 & $<0.001$ & Random \\
\hline$\geq 330$ & 4 & 1,609 & $1.31(1.08-1.60)$ & 0.007 & 54.2 & 0.088 & Random \\
\hline \multicolumn{8}{|l|}{ Treatment } \\
\hline Surgery & 5 & 1,915 & $1.67(1.15-2.41)$ & 0.007 & 92.2 & $<0.001$ & Random \\
\hline Surgery + chemotherapy/chemotherapy & 2 & 379 & $1.17(0.47-2.89)$ & 0.731 & 67.8 & 0.078 & Random \\
\hline \multicolumn{8}{|l|}{ Cut-off value } \\
\hline$\geq 2$ & 1 & 382 & $1.15(1.05-1.25)$ & 0.002 & - & - & - \\
\hline$\geq 3$ & 4 & 1,261 & $1.61(0.89-2.91)$ & 0.112 & 90.8 & $<0.001$ & Random \\
\hline$\geq 4$ & 2 & 651 & $1.67(1.26-2.20)$ & $<0.001$ & 0 & 0.842 & Fixed \\
\hline \multicolumn{8}{|l|}{ Survival analysis } \\
\hline MVA & 6 & 1,988 & $1.56(1.04-2.34)$ & 0.030 & 90.5 & $<0.001$ & Random \\
\hline UVA & 1 & 306 & $1.52(1.18-1.94)$ & 0.001 & - & - & - \\
\hline \multicolumn{8}{|l|}{ Recurrence-free survival } \\
\hline Total & 5 & 1,606 & $1.47(0.97-2.23)$ & 0.072 & 85.6 & $<0.001$ & Random \\
\hline \multicolumn{8}{|l|}{ Country } \\
\hline Japan & 4 & 1,312 & $1.23(0.95-1.59)$ & 0.121 & 50.7 & 0.107 & Random \\
\hline China & 1 & 294 & $2.93(2.10-4.09)$ & $<0.001$ & - & - & - \\
\hline \multicolumn{8}{|l|}{ Sample size } \\
\hline$<330$ & 3 & 673 & $1.83(1.03-3.25)$ & 0.039 & 78.6 & 0.009 & Random \\
\hline$\geq 330$ & 2 & 933 & $1.11(0.92-1.35)$ & 0.267 & 0 & 0.971 & Fixed \\
\hline \multicolumn{8}{|l|}{ Treatment } \\
\hline Surgery & 3 & 1,227 & $1.53(0.85-2.73)$ & 0.155 & 91.8 & $<0.001$ & Random \\
\hline Surgery + chemotherapy/chemotherapy & 2 & 379 & $1.37(0.65-2.85)$ & 0.406 & 67.9 & 0.078 & Random \\
\hline \multicolumn{8}{|l|}{ Cutoff value } \\
\hline$\geq 3$ & 3 & 955 & $1.47(0.69-3.13)$ & 0.318 & 90.7 & $<0.001$ & Random \\
\hline$\geq 4$ & 2 & 651 & $1.41(0.85-2.34)$ & 0.187 & 78.3 & 0.032 & Random \\
\hline
\end{tabular}

COUNT, controlling nutritional status; OS, overall survival; RFS, recurrence-free survival; MVA, multivariate analysis; UVA, univariate analysis.

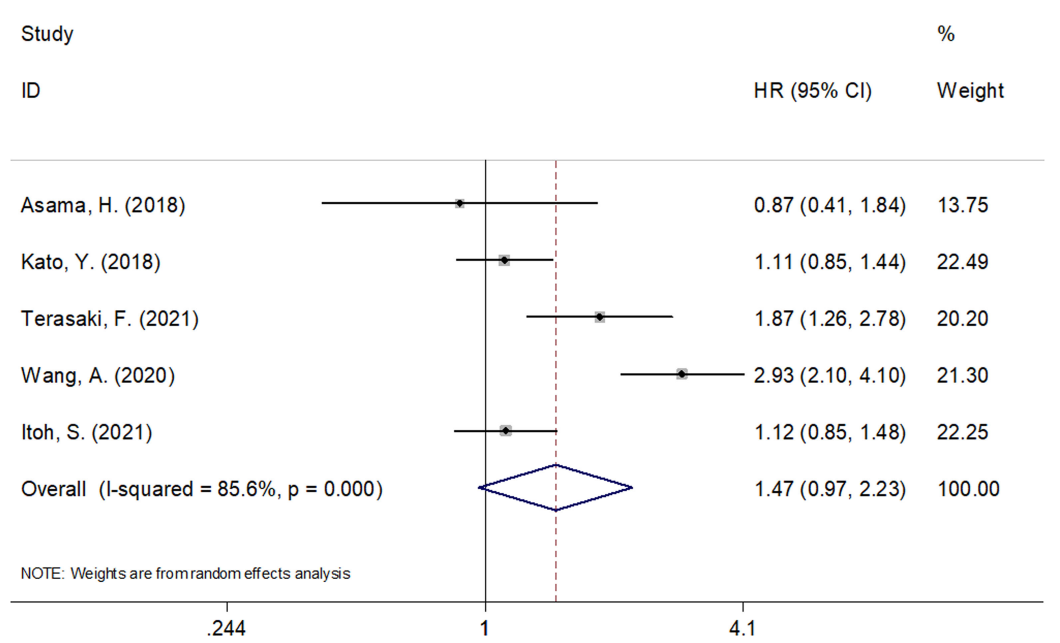

FIGURE 3 | Forest plots of CONUT score in predicting RFS in pancreatic cancer.

a poor RFS for Chinese patients with pancreatic cancer (HR = 2.93, 95\% CI $=2.10-4.09, p<0.001)$ and in studies with sample size $<330(\mathrm{HR}=1.83,95 \% \mathrm{CI}=1.03-3.25, p=0.039)$
(Table 3). However, the CONUT score was not significantly associated with RFS irrespective of treatment or the cutoff CONUT value. 


\section{Association Between CONUT Score and Patient Characteristics}

We investigated the correlation between the CONUT score and the clinicopathological characteristics of patients with pancreatic cancer using the data from five studies with 1,633 patients (10, 12-15). As shown in Table 4, a high CONUT score was significantly associated with male patients (OR $=1.34,95 \%$ $\mathrm{CI}=1.03-1.75, p=0.029)$. However, the association between the CONUT score and the clinical stage $(\mathrm{OR}=1.11,95 \% \mathrm{CI}=$ $0.46-2.71, \mathrm{p}=0.576)$, lymph node metastasis ( $\mathrm{OR}=0.73,95 \%$ $\mathrm{CI}=0.39-1.36, \mathrm{p}=0.323)$, lymphatic vessel invasion $(\mathrm{OR}=0.84$, $95 \% \mathrm{CI}=0.55-1.28, \mathrm{p}=0.411)$, invasion of the portal vein system $(\mathrm{OR}=1.04,95 \% \mathrm{CI}=0.51-2.13, \mathrm{p}=0.915)$, and nerve plexus invasion $(\mathrm{OR}=1.22,95 \% \mathrm{CI}=0.83-1.80, \mathrm{p}=0.318)$ in pancreatic cancer was not significant (Table 4).

\section{Publication Bias}

Potential publication bias was evaluated using Begg's test and Egger's test. Figure 4 shows there is no significant publication bias with respect to OS (Begg's test: $p=0.764$; Egger's test: $p=$ 0.717 ) and RFS (Begg's test: $p=0.463$, Egger's test: $p=0.792$ ).

\section{DISCUSSION}

In recent years, the prognostic value of inflammatory and nutritional indices of patients with cancer has been extensively investigated (19-21). Studies have investigated the prognostic value of the CONUT score for patients with pancreatic cancer, but the results were inconsistent. In our meta-analysis, we combined data from seven studies that comprised 2,294 patients. The pooled data demonstrated that a high CONUT score was a significant prognostic biomarker for predicting the OS but not the RFS of patients with pancreatic cancer. Additionally, an elevated CONUT score correlated with male patients with pancreatic cancer, which suggests that male patients with pancreatic cancer tend to have higher CONUT scores. Thus, a high CONUT score was a reliable prognostic factor for a poor OS for patients with pancreatic cancer and could be used to identify high-risk patients. To our knowledge, this meta-analysis was the first to investigate the prognostic value of the CONUT score of patients with pancreatic cancer.

The CONUT score was computed using serum albumin, total lymphocyte count, and total cholesterol values. Patients with low levels of these parameters have a low CONUT score. The biological mechanisms involved in the prognostic value of the CONUT score with respect to a poor OS for patients with pancreatic cancer are still not fully understood but could be explained by the following points. First, the serum albumin level is an important indicator of nutritional status and is regarded as an acute-phase protein that has a role in systemic inflammation (22). Pretreatment hypoalbuminemia correlates with poor survival of patients with cancer (23). Second, lymphocytes play a pivotal role in cell-mediated antitumor immune responses (24). Tumor-infiltrating lymphocytes are important components of antitumor activity and can induce cytotoxic cell death and inhibit tumor cell proliferation (25). Therefore, low lymphocyte counts can weaken immune responses and lead to poor survival for patients with cancer (26). Third, cholesterol is essential for the maintenance of cell membrane function, which is crucial for signal transduction. Decreased levels of cholesterol can affect the antitumor activity of immunocompetent cells (27). Therefore, a low CONUT score could represent a combination of a low serum albumin level, a low lymphocyte count, and a low total cholesterol level, which is reasonably associated with poor survival in patients with cancer.

Many recent meta-analysis studies also have investigated the prognostic role of the CONUT score with respect to solid tumors. In a meta-analysis that included 3,029 patients, Peng et al. showed that a high CONUT score positively correlated with poor prognoses in patients with non-small cell lung cancer (28). Another meta-analysis of six studies demonstrated that a high CONUT score correlated with a poor OS, cancer-specific survival, and DFS in patients with upper tract urothelial carcinoma or renal cell carcinoma undergoing nephrectomy (29). A meta-analysis by Takagi et al. showed that the preoperative CONUT score was an independent prognostic indicator of survival in patients with gastric cancer (30). Our meta-analysis investigated the prognostic efficiency of the CONUT score for patients with pancreatic cancer. We identified a positive association between the CONUT score and the OS of patients with pancreatic cancer, whereas its prognostic role with respect to the RFS was not significant, maybe because of the relatively short follow-up period for RFS analysis and the small sample size (only five studies were included in the RFS analysis). The prognostic value of the CONUT score for predicting the RFS in pancreatic cancer should be verified by future large-scale trials.

TABLE 4 | The association between COUNT score and clinicopathological features in patients with pancreatic cancer.

\begin{tabular}{|c|c|c|c|c|c|c|c|}
\hline \multirow[t]{2}{*}{ Factors } & \multirow[t]{2}{*}{ No. of studies } & \multirow[t]{2}{*}{ No. of patients } & \multirow[t]{2}{*}{ OR (95\% Cl) } & \multirow[t]{2}{*}{$\mathbf{p}$} & \multicolumn{2}{|c|}{ Heterogeneity } & \multirow[t]{2}{*}{ Effects model } \\
\hline & & & & & $I^{2}(\%)$ & $\mathbf{P h}$ & \\
\hline Sex (male vs. female) & 4 & 1,289 & $1.34(1.03-1.75)$ & 0.029 & 29.6 & 0.235 & Fixed \\
\hline Clinical stage (III-IV vs. |-II) & 4 & 1,289 & $1.11(0.46-2.71)$ & 0.576 & 85.7 & $<0.001$ & Random \\
\hline Lymph node metastasis (positive vs. negative) & 5 & 1,633 & $0.73(0.39-1.36)$ & 0.323 & 80.8 & $<0.001$ & Random \\
\hline Lymphatic vessel invasion (positive vs. negative) & 2 & 638 & $0.84(0.55-1.28)$ & 0.411 & 0 & 0.957 & Fixed \\
\hline Invasion of portal vein system (positive vs. negative) & 2 & 638 & $1.04(0.51-2.13)$ & 0.915 & 73.6 & 0.052 & Random \\
\hline Nerve plexus invasion (positive vs. negative) & 2 & 638 & $1.22(0.83-1.80)$ & 0.318 & 0 & 0.663 & Fixed \\
\hline
\end{tabular}

COUNT, controlling nutritional status. 

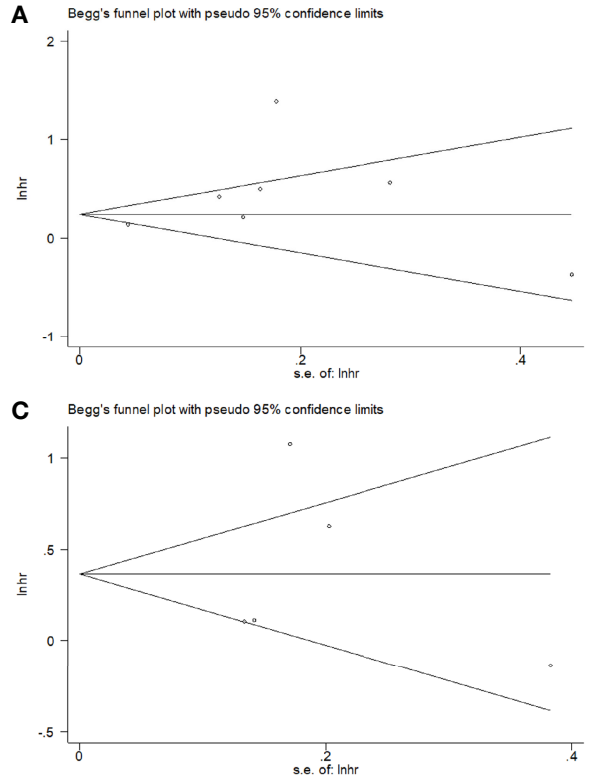
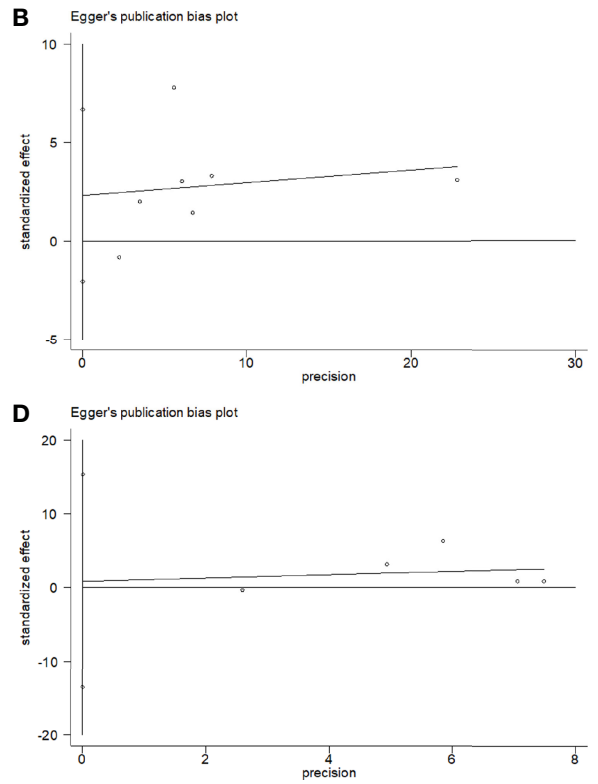

FIGURE 4 | Publication bias test by Begg's funnel plot and Egger's test. (A) Begg's test for OS, $p=0.764$; (B) Egger's test for OS, $p=0.717$; (C) Begg's test for RFS, $p=0.463$; (D) Egger's test for RFS, $p=0.792$.

This meta-analysis has several limitations. First, the sample size was relatively small. Only seven studies with 2,294 patients were included in the analysis. The small sample size may have introduced a selection bias in this meta-analysis because when the sample size is small, the data of each individual could have a greater impact on the overall results than when the sample size is larger (31). Second, all included studies were conducted in Asian countries, namely, Japan and China. Although we thoroughly searched the literature, no studies of non-Asian patients met our inclusion criteria. Third, the included studies did not have the same cutoff CONUT scores; four studies used $\geq 3$, two studies used $\geq 4$, and one study used $\geq 2$. The different cutoff CONUT scores may have caused the heterogeneity among the studies.

\section{CONCLUSIONS}

In summary, our meta-analysis showed that a high CONUT score is significantly associated with a poor OS for patients with

\section{REFERENCES}

1. Bray F, Ferlay J, Soerjomataram I, Siegel RL, Torre LA, Jemal A. Global Cancer Statistics 2018: GLOBOCAN Estimates of Incidence and Mortality Worldwide for 36 Cancers in 185 Countries. CA: Cancer J Clin (2018) 68 (6):394-424. doi: 10.3322/caac.21492

2. Hu JX, Zhao CF, Chen WB, Liu QC, Li QW, Lin YY, et al. Pancreatic Cancer: A Review of Epidemiology, Trend, and Risk Factors. World J Gastroenterol (2021) 27(27):4298-321. doi: 10.3748/wjg.v27.i27.4298

3. Bengtsson A, Andersson R, Ansari D. The Actual 5-Year Survivors of Pancreatic Ductal Adenocarcinoma Based on Real-World Data. Sci Rep (2020) 10(1):16425. doi: 10.1038/s41598-020-73525-y pancreatic cancer. Male patients with pancreatic cancer tend to have a higher CONUT score. The CONUT score may be an effective prognostic factor in pancreatic cancer in clinical practice.

\section{DATA AVAILABILITY STATEMENT}

All datasets generated for this study are included in the article/ supplementary material. Further inquiries can be directed to the corresponding author.

\section{AUTHOR CONTRIBUTIONS}

$\mathrm{XM}$ and WZ collected, extracted, and performed the quality assessment and analyzed the data; YS conceived and designed this study and wrote the paper. All authors reviewed the final manuscript. All authors contributed to the article and approved the submitted version. 
7. Xu J, Shi KQ, Chen BC, Huang ZP, Lu FY, Zhou MT. A Nomogram Based on Preoperative Inflammatory Markers Predicting the Overall Survival of Pancreatic Ductal Adenocarcinoma. J Gastroenterol Hepatol (2017) 32 (7):1394-402. doi: 10.1111/jgh.13676

8. Imaoka H, Mizuno N, Hara K, Hijioka S, Tajika M, Tanaka T, et al. Evaluation of Modified Glasgow Prognostic Score for Pancreatic Cancer: A Retrospective Cohort Study. Pancreas (2016) 45(2):211-7. doi: 10.1097/mpa. 0000000000000446

9. Asama H, Suzuki R, Takagi T, Sugimoto M, Konno N, Watanabe K, et al. Evaluation of Inflammation-Based Markers for Predicting the Prognosis of Unresectable Pancreatic Ductal Adenocarcinoma Treated With Chemotherapy. Mol Clin Oncol (2018) 9(4):408-14. doi: 10.3892/mco. 2018.1696

10. Kato Y, Yamada S, Suenaga M, Takami H, Niwa Y, Hayashi M, et al. Impact of the Controlling Nutritional Status Score on the Prognosis After Curative Resection of Pancreatic Ductal Adenocarcinoma. Pancreas (2018) 47(7):8239. doi: $10.1097 / \mathrm{mpa} .0000000000001105$

11. Ignacio de Ulíbarri J, González-Madroño A, de Villar NG, González P, González B, Mancha A, et al. CONUT: A Tool for Controlling Nutritional Status. First Validation in a Hospital Population. Nutr Hosp (2005) 20 (1):38-45.

12. Mao YS, Hao SJ, Zou CF, Xie ZB, Fu DL. Controlling Nutritional Status Score is Superior to Prognostic Nutritional Index Score in Predicting Survival and Complications in Pancreatic Ductal Adenocarcinoma: A Chinese Propensity Score Matching Study. Br J Nutr (2020) 124(11):1190-7. doi: 10.1017/ s0007114520002299

13. Terasaki F, Sugiura T, Okamura Y, Ito T, Yamamoto Y, Ashida R, et al. The Preoperative Controlling Nutritional Status (CONUT) Score is an Independent Prognostic Marker for Pancreatic Ductal Adenocarcinoma. Updates Surg (2021) 73(1):251-9. doi: 10.1007/s13304-020-00792-9

14. Wang A, Sun B, Wang M, Shi H, Huang Z, He T, et al. Predictive Value of CONUT Score Combined With Serum CA199 Levels in Postoperative Survival of Patients With Pancreatic Ductal Adenocarcinoma: A Retrospective Study. PeerJ (2020) 8:e8811. doi: 10.7717/peerj.8811

15. Dang C, Wang M, Zhu F, Qin T, Qin R. Controlling Nutritional Status (CONUT) Score-Based Nomogram to Predict Overall Survival of Patients With Pancreatic Cancer Undergoing Radical Surgery. Asian J Surg (2021). doi: 10.1016/j.asjsur.2021.08.011

16. Itoh S, Tsujita E, Fukuzawa K, Sugimachi K, Iguchi T, Ninomiya M, et al. Prognostic Significance of Preoperative PNI and CA19-9 for Pancreatic Ductal Adenocarcinoma: A Multi-Institutional Retrospective Study. Pancreatology (2021) 21(7):1356-63. doi: 10.1016/j.pan.2021.08.003

17. Moher D, Liberati A, Tetzlaff J, Altman DG, Grp P. Preferred Reporting Items for Systematic Reviews and Meta-Analyses: The PRISMA Statement. PloS Med (2009) 6(7):e1000097. doi: 10.1371/journal.pmed.1000097

18. Stang A. Critical Evaluation of the Newcastle-Ottawa Scale for the Assessment of the Quality of Nonrandomized Studies in Meta-Analyses. Eur J Epidemiol (2010) 25(9):603-5. doi: 10.1007/s10654-010-9491-z

19. Greten FR, Grivennikov SI. Inflammation and Cancer: Triggers, Mechanisms, and Consequences. Immunity (2019) 51(1):27-41. doi: 10.1016/j.immuni.2019.06.025

20. Ravasco P. Nutrition in Cancer Patients. J Clin Med (2019) 8(8):1211. doi: $10.3390 / \mathrm{jcm} 8081211$

21. Mitchell T, Clarke L, Goldberg A, Bishop KS. Pancreatic Cancer Cachexia: The Role of Nutritional Interventions. Healthcare (2019) 7(3):89. doi: 10.3390/healthcare7030089
22. Caraceni P, Tufoni M, Bonavita ME. Clinical Use of Albumin. Blood Transfus = Transfus Sangue (2013) 11 Suppl 4(Suppl 4):s18-25. doi: 10.2450/2013.005s

23. Gupta D, Lis CG. Pretreatment Serum Albumin as a Predictor of Cancer Survival: A Systematic Review of the Epidemiological Literature. Nutr J (2010) 9:69. doi: 10.1186/1475-2891-9-69

24. Bastid J, Bonnefoy N, Eliaou JF, Bensussan A. Lymphocyte-Derived Interleukin-17A Adds Another Brick in the Wall of Inflammation-Induced Breast Carcinogenesis. Oncoimmunology (2014) 3:e28273. doi: 10.4161/ onci. 28273

25. Minami T, Minami T, Shimizu N, Yamamoto Y, De Velasco M, Nozawa M, et al. Identification of Programmed Death Ligand 1-Derived Peptides Capable of Inducing Cancer-Reactive Cytotoxic T Lymphocytes From HLA-A24+ Patients With Renal Cell Carcinoma. J Immunother (Hagerstown Md: 1997) (2015) 38(7):285-91. doi: 10.1097/cji.0000000000000090

26. Väyrynen JP, Tuomisto A, Klintrup K, Mäkelä J, Karttunen TJ, Mäkinen MJ. Detailed Analysis of Inflammatory Cell Infiltration in Colorectal Cancer. $\mathrm{Br}$ J Cancer (2013) 109(7):1839-47. doi: 10.1038/bjc.2013.508

27. Chimento A, Casaburi I, Avena P, Trotta F, De Luca A, Rago V, et al. Cholesterol and Its Metabolites in Tumor Growth: Therapeutic Potential of Statins in Cancer Treatment. Front Endocrinol (Lausanne) (2018) 9:807. doi: 10.3389/fendo.2018.00807

28. Peng J, Hao Y, Rao B, Cao Y. Prognostic Impact of the Pre-Treatment Controlling Nutritional Status Score in Patients With Non-Small Cell Lung Cancer: A Meta-Analysis. Med (Baltimore) (2021) 100(26):e26488. doi: $10.1097 / \mathrm{md} .0000000000026488$

29. Chen J, Cao D, Peng Z, Song P, Liu Z, Yang L, et al. The Prognostic Value of the Controlling Nutritional Status (CONUT) Score on Patients Undergoing Nephrectomy for Upper Tract Urothelial Carcinoma or Renal Cell Carcinoma: A Systematic Review and Meta-Analysis. Br J Nutr (2021), 120. doi: $10.1017 / \mathrm{s} 0007114521002889$

30. Takagi K, Domagala P, Polak WG, Buettner S, Wijnhoven BPL, Ijzermans JNM. Prognostic Significance of the Controlling Nutritional Status (CONUT) Score in Patients Undergoing Gastrectomy for Gastric Cancer: A Systematic Review and Meta-Analysis. BMC Surg (2019) 19(1):129. doi: 10.1186/s12893019-0593-6

31. Sundberg R. Small Sample and Selection Bias Effects in Calibration Under Latent Factor Regression Models. J Chemometr (2007) 21(5-6):227-38. doi: $10.1002 / \mathrm{cem} .1064$

Conflict of Interest: The authors declare that the research was conducted in the absence of any commercial or financial relationships that could be construed as a potential conflict of interest.

Publisher's Note: All claims expressed in this article are solely those of the authors and do not necessarily represent those of their affiliated organizations, or those of the publisher, the editors and the reviewers. Any product that may be evaluated in this article, or claim that may be made by its manufacturer, is not guaranteed or endorsed by the publisher.

Copyright $\odot 2022 \mathrm{Ma}$, Zou and Sun. This is an open-access article distributed under the terms of the Creative Commons Attribution License (CC BY). The use, distribution or reproduction in other forums is permitted, provided the original author(s) and the copyright owner(s) are credited and that the original publication in this journal is cited, in accordance with accepted academic practice. No use, distribution or reproduction is permitted which does not comply with these terms. 